\title{
A Review Report on Time Domain-Attribute-based Access Control for Video substance distribution: A Cryptographic Approach
}

\author{
Kumar Gaurav \\ PhD Scholar \\ Department of E.C.E. \\ AISECT University, Bhopal
}

\author{
Sanjeev Gupta, PhD \\ Professor, Department of E.C.E \\ AISECT University, Bhopal
}

\begin{abstract}
Visual cryptography encodes a secret binary image (SI) keen on share of unsystematic binary sample. If the share is xeroxed against transparencies, the key figure is often visually decrypted by means that of superimposing a skilled subset of transparencies, however no secret data is obtained from the superposition of a forbidden subset. The binary model of the $\mathrm{N}$ allocates, although, contain no visual consequence and hinder the objectives of visual cryptography. The perfect security condition of $\mathrm{VC}$ scheme needs the strict demand where any $\mathrm{t}-1$ or fewer transparencies cannot extract much knowledge regarding the key. The secret image is at the same time embedded into color halftone shares. Visual cryptography (VC) could be a secret sharing method of decay a secret picture into $n$ transparencies, and consequently the stack of any $t$ out of $n$ transparencies disclose the key content. A HVC generate procedure is projected that can make an option for secret halftone image into color half-tone shares. In [1], authors centre of attention how to securely distribute video contents to an assured collection of persons for the period of a testing time period in cloud-based multimedia systems, and propose a cryptographic come within reach of, a provably secure time domain attribute-based access control (TAAC) scheme, to secure the cloud-based video content sharing.
\end{abstract}

\section{Keywords}

Cloud computing, multimedia, time-domain, TAAC (timedomain attribute-based access control), content video sharing.

\section{INTRODUCTION}

Internet of Things (IoT) has appeared as a persistent communications of the information society that enables physical sensors, smart phones and smart buildings to interconnect with each other. With the rapid development of wireless technologies and mobile devices, mobile social networks (MSNs) have been widely used in daily life, where mobile users can obtain the desired social services conveniently [3]. In the era of big data, a huge amount of data can be generated quickly from various sources (e.g., smart phones, sensors, machines, social networks, etc.). The demands on video quality and user experience have also been increasing significantly in many video applications, such as Ultra-high definition (UHD) live streaming, 3-D movies, instant high definition (HD) video messages, etc [1]. When the cloud server is in use as the dealer, the privacy issue becomes much more critical in information publish-subscribe systems as the cloud server cannot be fully trusted. Specifically, there are three major privacy requirements: 1) Data Privacy. The cloud server and other unauthorized users are not allowed to access the published data; 2) Tag Privacy. The tags associated with the data should not reveal the keywords that may indicate the data content; and 3) Trapdoor Privacy. The subscription trapdoors should not reveal any keywords or the subscription policy that may indicate the interests of the subscribers. VISUAL cryptography (VC) may possibly be a division of secret sharing information. In the VC idea, a secret image is encoded into transparencies, and furthermore the content of each transparency is noise-like in order to the secret information cannot be retrieved from anyone clearness via human visual scrutiny or signal study techniques. In general, a -threshold VC theme has the subsequent properties: The stacking of any out of these VC generated transparencies will reveal the secret by perception, however the stacking of any or fewer variety of transparencies cannot retrieve any data other than the dimensions of the secret image. Naor and Shamir [17] planned a -threshold VC theme based on basis matrices, and also the model had been more considered and extended. The connected mechanism include the VC method maintain on probabilistic representation, frequent access configuration, $\mathrm{VC}$ over halftone photo, $\mathrm{VC}$ for color photo, cheating in $\mathrm{VC}$, the concluding formula of $\mathrm{VC}$ schemes, and region incrementing VC. Contrast is one altogether the necessary performance metrics for $\mathrm{VC}$ schemes. Generally, the stacking revelation of the key with higher contrast represents the higher visual quality, and thus the stacking secret with high contrast is that the goal of pursuit in VC designs. Naor and Shamir [17] define a contrast formula that has been wide utilized in several studies. Based on the definition of contrast, there are studies attempting to achieve the contrast certain of VC theme. For example, Blundo et al. [21] provides the optimal contrast of VC schemes. Krause and Simon et al. [9] offers a linear program that is able to calculate precisely the optimal contrast for $\mathrm{VC}$ schemes. Krause and Simon [9] offer the upper bound 
and lower bound of the optimum contrast for $\mathrm{VC}$ schemes. Moreover, there exist VC connected researches exploitation differential definitions of contrast. A different required metric is that the pixel expansion denoting the amount of sub pixels in transparency used to encode a secret pixel. The minimization of pixel expansions has been investigated in previous studies. The probabilistic model of the VC theme was 1 st introduced, wherever the theme is based on the idea matrices, however only 1 column of the matrices is chosen to encode a binary secret pixel, instead of the normal VC theme utilizing the entire basis matrices. The dimension of the generated transparencies is identical to the secret image. Yang [8] furthermore planned a probabilistic model of $\mathrm{VC}$ scheme and also the 2 cases and is clearly made to achieve the optimum contrast. Based on principle, Cimatoe planned a generalized VC theme within which the pixel expansion is between the probabilistic model of VC theme and also the traditional VC theme. Encrypting a picture by random grids (RGs) was initial introduced by Kafri and Keren [16] in 1987. A binary secret picture is set into 2 noise-like transparencies with a like size of the original secret picture, and mountain of the 2 transparencies reveals the pleased of the secret. Evaluation of RGs with source atmosphere, one amongst the major benefits is that the measurement of produce transparencies is un-expanded. The RG premise is like to the probabilistic representation of the VC design; however the RG premise isn't support on the basis matrices. The current studies consist of the RG for colour image, $R G$, and $R G$ schemes. We tend to furthermore evaluate the designed method with RG.

\section{LITERATURE SURVEY}

Kan Yang et. al. [1] "Time-Domain Attribute-Based Access Control for Cloud-Based Video Content Sharing: A Cryptographic Approach" through the ever-increasing demands on multimedia system applications, cloud computing, because of its economical however powerful resources, is turning into a natural platform to method, store, and share multimedia system contents. However, the use of cloud computing additionally brings new security and privacy problems as few public cloud servers are totally sure by users. During this paper, we tend to specialize in a way to securely share video contents to a particular group of people throughout a specific period of time in cloud-based multimedia system systems, and propose a cryptographic approach, a provably secure time domain attribute-based access control (TAAC) scheme, to secure the cloud-based video content sharing. Specifically, we tend to 1 st propose a provably secure time-domain attribute-based encryption scheme by embedding the time into each the cipher texts and therefore the keys, specified only users WHO hold sufficient attributes during a specific time interval will decode the video contents. We tend to additionally propose an efficient attribute change methodology to realize the dynamic modification of users' attributes, as well as granting new attributes, revoking previous attributes, and regrating previously revoked attributes.

Vilma Petrauskiene et .al. [2] "Dynamic visual cryptography for optical assessment of chaotic oscillations" An optical experimental technique based on dynamic visual cryptography is proposed for the optical assessment of chaotic oscillations. The secret image is embedded into a single cover image which is fixed onto the surface of the oscillating structure. It is demonstrated that this visual scheme is applicable for the assessment of chaotic oscillation seven though time-average dmoiré fringes do not form when the encoded cover image is oscillated by the chaotic law.

Zhila Bahrami et. al. [3] "A new robust video watermarking algorithm based on SURF features and block classification" In this author planned strong block classification primarily based semi-blind video watermarking algorithmic rule using visual cryptography and SURF (Speed-Up robust Features) options to enhance the lustiness, stability, imperceptibility and time period performance. Technique of choosing the simplest frames in every shot and therefore the best regions or blocks at intervals best frames is planned to avoid using frame-byframe method for generating owner's share so as to enhance robustness furthermore as reducing time complexness. In our methodology, Owner's share is generated using the classification of selected strong blocks at intervals the chosen frames alongside corresponding watermark data.

Paulius Palevicius et. al. [4] "Image communication scheme based on dynamic visual cryptography and computer generated holography" This paper proposes the mixture of dynamic visual cryptography (an optical technique supported the interaction of visual cryptography and time-averaging geometric moiré) with Gerchberg-Saxton algorithmic rule. A stochastic moiré grating is employed to plant the secret into one cover image. The secret might even be visually decoded by an academe if only the amplitude of harmonic oscillations corresponds to an accurately preselected value. The planned visual image encryption scheme is predicated on computer generated holography, an image hiding scheme supported pc generated holography and dynamic visual cryptography is projected throughout this paper. The key image is embedded into the random geometric moiré cover image. GerchbergSaxton algorithmic rule is employed to produce part info from the encrypted cover image and is directly incorporated into $\mathrm{CGH}$. the key image is leaked from time-averaged pattern of fringes generated by an oscillatory $\mathrm{CGH}$ image inside the projection plane; the decoding is totally optical and does not want an application of a computing device.

Anjney Pandey et. al. [5] "Applications and Usage of Visual Cryptography: A Review" In this paper, we tend to shall study the various application areas of Visual Cryptography. Visual Cryptography could be a wide space of analysis utilized in information hiding, securing pictures, color imaging, multimedia system and different such fields. Visual Cryptography comes within the field of information hiding utilized in cybercrime, file formats etc. This paper focuses on the application areas of visual cryptography from four totally different analysis papers/journals that mention the most important application areas of visual cryptography. Applications of Visual Cryptography talked about during this review paper focus primarily on the utilization of coding that is the most important feature of visual cryptography. The analysis papers reviewed on top of describe one in every of the simplest applications and aspects of visual cryptography.

Lifeng Yuan et. al. [6] "Secret Image Sharing Scheme with Threshold Changeable Capability" In secret image sharing schemes, the edge would possibly got to be adjusted simply in case of changes at intervals the safety policy and so the adversary structure before recovering the key image. As an example, if participants leave the group, their stego pictures 
are useless to them and cannot be kept safely. As a result, these pictures are usually simply stolen and utilized by intruders that reduce the protection of the scheme. To resolve this disadvantage, we tend to tend to propose a very unique threshold changeable secret image sharing scheme with $N$ potential changeable thresholds $t 1, t 2 \ldots t N$. By preparing advance shares for thresholds $t 1, t 2 \ldots t N$ and using the twovariable one-way perform to return up with the identification value; we are going to modification the threshold once necessary. Before recovering the key image, the edge may got to be compelled to be adjusted for the modification of the safety policy and so the adversary's structure.

MA Zhaofeng et. al. [7] “A Novel Image Digital Rights Management Scheme with High-Level Security, Usage Control and Traceability" in projected work a unique image digital rights management scheme for Confidential image information security supported encryption and watermark (CIDSEW) with high-level security, usage control and traceability, within which we tend to used full content image encryption for confidentiality of the image to be protected, and that we first projected strict and elaborate Usage control (UC) scheme for Confidential image information (CIData) usage password-based authentication, opening times, printing and exporting control. And once the CIData got to delivery or export to different users or domain, we tend to projected the secure export and misused tracing and observe approach, within which before the ciphered CI information is exported, we tend to decrypted the CI information in a very plain mode, and simultaneously we tend to embedded user-identity-related and hardware-related data as strong watermark for traceability and responsibility confirmation. during this paper, we tend to projected a unique image digital rights management scheme for Confidential Image information Security supported encryption and Watermark (CIDSEW) with high-level security, usage control and traceability, within which we tend to used full content image encryption for confidentiality of the image to be protected, and that we first projected strict and detailed Usage control (UC) scheme for Confidential image information (CI Data) usage password primarily based authentication, opening times, printing and exporting control.

Xiuli Chai et. al. [8] "A new chaos-based image encryption algorithm with dynamic key selection mechanisms" In recent years, a wide reasonably cryptographically algorithms supported chaos has been suggests and most of them are proved to realize success by adopting the standard permutation-diffusion style. However, one disadvantage these methods principally hold is that they have little reference to the plaintext or, properly speaking, the connection between them is kind of less. The disadvantage makes the coding algorithms vulnerable to the known-plaintext and chosenplaintext attack. Additionally, the key keys are stationary at the most times, which they can't be selected dynamically by the corresponding plain image pixels. Therefore on overcome these disadvantages mentioned on top of, we tend to introduce a current chaos-based image encoding algorithmic rule with dynamic key selection mechanisms during this paper, and present a dynamic key stream sequence group selection mechanism (DKSGSM) and a dynamic key stream selection mechanism (DKSM).

\section{METHOD}

\subsection{Normal or Body Text}

The work is essentially on Visual cryptography theme at intervals that half tone is applied .The most necessary aim to code transparencies and additionally the content of each transparency is noise like thus secret information can't be retrieved from anyone transparency via human visual observation or signal analysis. The initial color image is taken and three basic colours (red, green, blue) are extracted out it. Computer creates the colors supported RGB model shown in fig.2. It produces spectrum of visible light. Monitor can produce many colors by combining wholly completely different percentages of three primaries, red, green and blue. Whereas using the image process software system like Photoshop you'll see that these RGB colors are added with the help of numerical price, that's between 0 to 255 . With RGB, mixture of red and green equally provides yellow, mixture of green and blue creates cyan and additionally the combination of red and blue creates magenta. Once all the three colors, red, green and blue are mixed equally they produces white lightweight. Thus it's called Additive color model. Another RGB model based example is human eye itself and scanners. The essential advantage of RGB model is; it's useful for full color editing because it's big range of colors. But at an identical time this model is said to device dependent. It means the strategy colors showed on the screen depends on the hardware used to show it. For the extraction of red color the other two colors i.e., green and blue are made null, equally for the extraction of green color, blue and red color are created null so on. When extraction RGB is regenerate into CMY (cyan, magenta, yellow) that will be an opposite model of RGB. Printing inks are supported this model. With the whole presence of cyan, magenta and yellow we tend to tend to induce black. But much at intervals the printing industry it is not possible to create black with these three colors. The results of the mixture of CMY are muddy brown due to the impurities of the printing inks. Therefore black ink is added to induce solid black. The results of this technique CMYK model and $\mathrm{k}$ indicate black color that's in addition recognized as 'key' color. Since black could also be a full presence of color, you'll got to subtract the number of cyan, magenta and yellow to supply the lighter colors. This might be explained in many strategies. Once light-weight falls on the green surface or green ink it absorbs (subtracts) all the colors from lightweight except green. Therefore the model is called subtractive model. Print production depends on this model.

It is helpful to possess correct understanding of the color models. The monitors additionally as scanner works on RGB principle. Whereas scanning we'll modification the software system to produce desired result. CMYK is for print business. It cannot manufacture the color vary of RGB therefore once finishing the work on laptop in RGB mode once you convert it into CMYK for printing some tonal changes is occurred. In spite of its limitation CMYK model is considered as best model offered for printing as results of it'll manufacture properly finished output.

Conversion of cyan color is completed by subtracting green and blue color from the most image pixel whereas conversion of magenta is completed by subtracting red and green color by from the most image pixel and then on shown in fig 2 .

When the image is converted into CMY, it's combined into one image and Floyd halftone is applied specifically shown in fig.1.Halftone is that the reprographic technique that simulates continuous tone imaging through the use of dots, variable either in size or in spacing, so generating a gradient like impact. "Halftone" will even be accustomed refer specifically to the image that is created by this technique. In which uninterrupted tone imaging include an infinite differ of colors or greys, the halftone procedure reduce illustration reproduction to an representation that is write down with only 
one color of ink, in dots of differing size (amplitude modulation) or spacing (frequency modulation). This reproduction depends on a basic optical illusion: the little halftone dots are blended into smooth tones by the human eye.

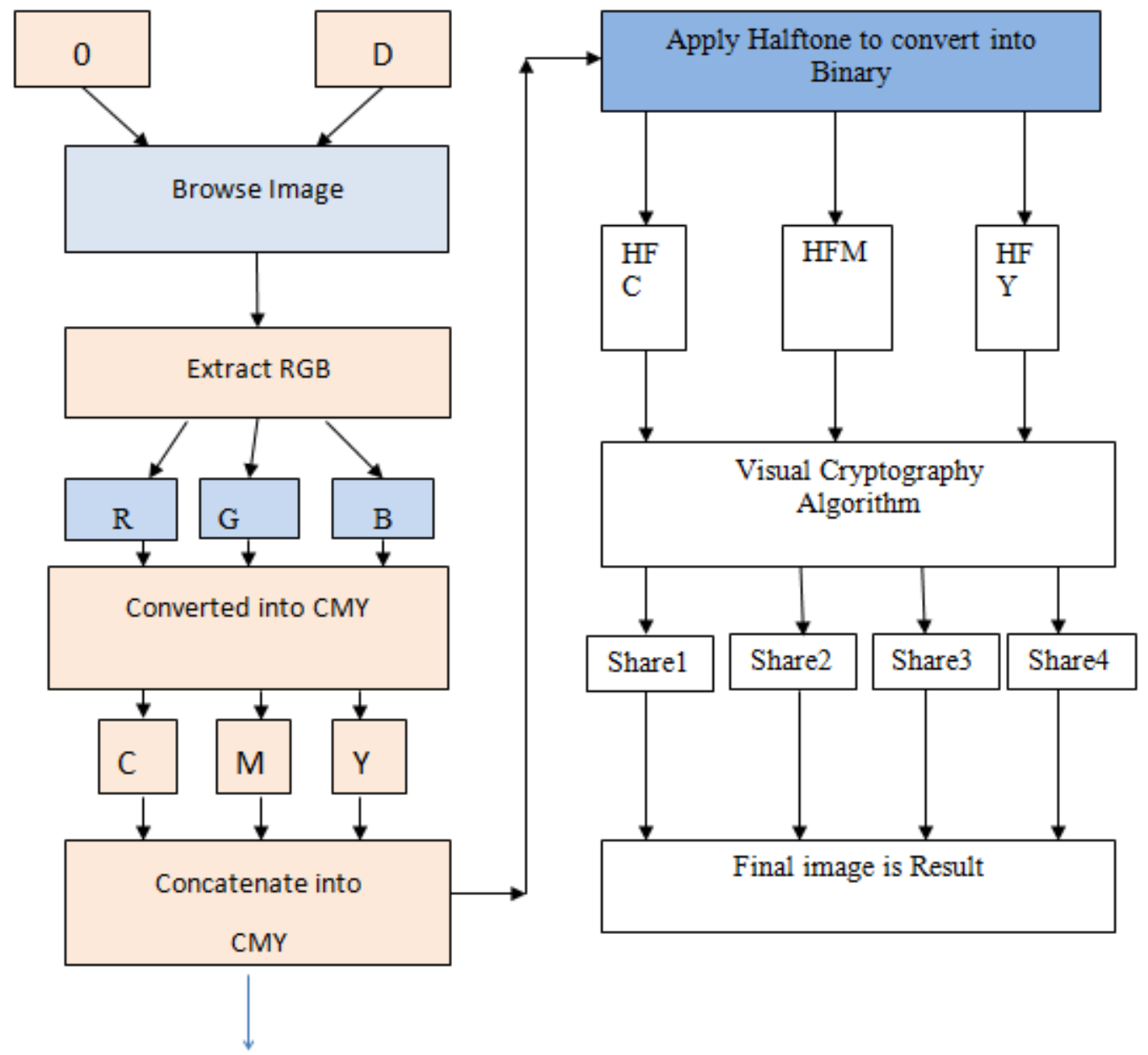

Figure 1: Proposed Methodology

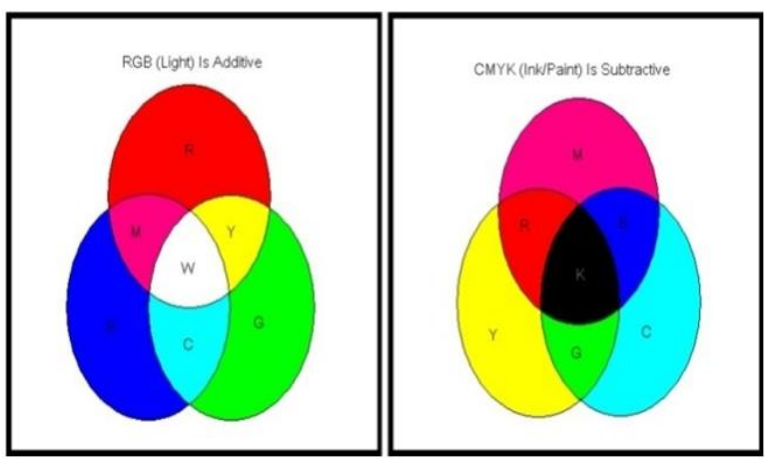

Figure 2: Van diagram for colour Conversion

At a microscopic level, developed black-and-white photographic material in addition consists of only two colours, and not an infinite vary of continuous tones.

After applying halftone to the image visual cryptography algorithms are done in order to make secret shares. One altogether the known technique has been credited to MoniNaor and Adi Shamir, WHO developed it in 1994. They demonstrated a visible secret sharing theme, where an image was broken up into $\mathrm{n}$ shares thus only somebody with all $\mathrm{n}$ shares could decrypt the image, whereas any $\mathrm{n}$ - one shares revealed no information concerning the primary image. each share was written on a separate transparency, and decryption was performed by overlaying the shares. Once all $n$ shares were overlaid, the initial image would appear. There are several generalizations of the essential scheme also as k-outof-n visual cryptography. using a similar arrange, transparencies is also used to implement a one-time pad coding, where one transparency is also a shared random pad, and another transparency acts because the cipher text. Normally, there is an expansion of space demand in visual cryptography. but if one altogether the two shares is structured recursively, the efficiency of visual cryptography are highly increased. Finally merging all the shares will reveal the key image.

\section{CONCLUSION}

In this paper we tend to simply review some work connected on a crypto logical Approach for Attribute-Based Access control for Cloud-Based Video Content Sharing. During this study some results are given out like in [1] authors planned a cryptographic approach, TAAC, to achieve time-domain attribute-based access control for cloud-based video content sharing. Specifically, we've planned a provably secure timedomain $\mathrm{ABE}$ scheme by embedding the time into each the 
cipher texts and also the keys, specified only users WHO hold sufficient attributes during a specific period of time will decode the information. In [2] Dynamic visual cryptography has been with success used for optical control of vibration generation equipment. This whole-field non-destructive zeroenergy methodology may be effectively exploited for optical assessment of various vibrating structures. The essential plan of this optical assessment technique relies on the very fact that refined process tools are needed to encode the key image, however the decoding method is totally visual.

\section{REFERENCES}

[1] Y. Kan, et al. "Time-domain attribute-based access control for cloud-based video content sharing: A cryptographic approach" IEEE Trans. on Multi. 18.5 (2016): 940-950.

[2] P. Vilma, et al. "Dynamic visual cryptography for optical assessment of chaotic oscillations" Opt. \& Laser Tech. 57 (2014): 129-135.

[3] Bahrami, Zhila, and Fardin Akhlaghian Tab. "A new robust video watermarking algorithm based on SURF features and block classification" Mult. Tools and App. (2016): 1-19.

[4] P. Paulius, and M.Ragulskis. "Image communication scheme based on dynamic visual cryptography and computer generated holography" Opt. Comm. 335 (2015): 161-167.

[5] Pandey, Anjney, and Subhranil Som. "Applications and usage of visual cryptography: A review" Reliability, Infocom Technologies and Optimization (Trends and Future Directions)(ICRITO), 2016 5th International Conference on. IEEE, 2016.

[6] Yuan, Lifeng, et al. "Secret Image Sharing Scheme with Threshold Changeable Capability" Mathematical Problems in Engineering 2016 (2016).

[7] Ma, Zhaofeng, et al. "A Novel Image Digital Rights Management Scheme with High-Level Security, Usage Control and Traceability" Chinese Journal of Electronics 25.3 (2016): 481-494

[8] Chai, Xiuli, Kang Yang, and Zhihua Gan. "A new chaosbased image encryption algorithm with dynamic key selection mechanisms" Multimedia Tools and Applications (2016): 1-21.

[9] Hofmeister T., Krause M., and Simon H. U. "Contrastoptimal out of secret sharing schemes in visual cryptography" Comput. Sci., vol. 240, no. 2, pp. 471485, Jun. 2000.

[10] P. A. Eisen and D. R. Stinson "Threshold visual cryptography schemes with specified whiteness levels of reconstructed pixels" Designs Cryptography, vol. 25, no. 1, pp. 15-61, 2002.
[11] M. Nakajima and Y. Yamaguchi, "Extended visual cryptography for natural images" WSCG J., vol. 10, no. 2, pp. 303-310, 2002.

[12] H. Koga "A general formula of the -threshold visual secret sharing scheme" in Proc. Int. Theory and Application of Cryptology and Information Security: Advances in Cryptology, Dec. 2002, pp. 328-345.

[13] S. Cimato, R. De Prisco, and A. De Santis, "Probabilistic visual cryptography schemes" Computer J., vol. 49, no. 1, pp. 97-107, Jan. 2006.

[14] G. B. Horng, T. G. Chen, and D. S. Tsai, "Cheating in visual cryptography” Designs, Codes, Cryptography, vol. 38, no. 2, pp. 219-236, Feb. 2006.

[15] N. Macon and A. Spitzbart, "Inverses of Vandermonde matrices" Amer. Math. Monthly, vol. 65, no. 2, pp. 95100, Feb. 1958

[16] O. Kafri and E. Kerens, "Encryption of pictures and shapes by random grids" Letter of , vol. 12, no. 6, pp. 377-379, Jun. 1987.

[17] M. Naor and Shamir A. "Visual cryptography" in Advances Proc. in Cryptography 1995, vol. 950, LNCS, pp. 1-12.

[18] Ateniese G. Blundo C. A. De Santis, and D. R. Stinson, "Visual cryptography for general access structures" Inf. Computat., vol. 129, no. 2, pp. 86-106, Sep. 1996.

[19] E. R. Verheul and H. C. A. Van Tilborg, "Constructions and properties of out of visual secret sharing schemes" Designs Cryptography, vol. 11, no. 2, pp. 179-196, May 1997.

[20] C.C. Wu, L.H. Chen, "A Study on Visual Cryptography" National Chiao Tung University, Taiwan, R.O.C., 1998.

[21] C. Blundo, A. De Santis, and D. R. Stinson, "On the contrast in visual cryptography schemes" J. Cryptology, vol. 12, no. 4, pp. 261-289, 1999.

[22] R. Ito, H. Kuwakado, and H. Tanaka, "Image size invariant visual cryptography" IEICE Trans. Fundam. Electron. Commun. \& Comput. Sci., vol. 82, pp. 2172 2177, Oct. 1999.

[23] Weir, Jonathan, and WeiQi Yan. "Sharing multiple secrets using visual cryptography." Circuits and Systems, 2009. ISCAS 2009. IEEE International Symposium on. IEEE, 2009.

[24] Lee, Kai-Hui, and Pei-Ling Chiu. "An extended visual cryptography algorithm for general access structures." ieee transactions on information forensics and security 7.1 (2012): 219-229.

[25] Yan, Xuehu, et al. "Halftone visual cryptography with minimum auxiliary black pixels and uniform image quality." Digital Signal Processing 38 (2015): 53-65. 